PROCEEDINGS OF THE AMERICAN MATHEMATICAL SOCIETY

Volume 124, Number 2, February 1996

\title{
REMOVABILITY OF THE SINGULAR SET OF THE HEAT FLOW OF HARMONIC MAPS
}

\author{
YUNMEI CHEN AND LIVIO FLAMINIO
}

(Communicated by Peter Li)

\begin{abstract}
We show that the singular set of a weak stationary solution $u$ of the heat flow of harmonic maps between Riemannian manifolds $M$ and $N$, with $N$ compact, is removable if it has "parabolic codimension" greater than two and the initial energy $E\left(u_{0}\right)$ is sufficiently small.
\end{abstract}

\section{INTRODUCTION AND PRELIMINARIES}

Let $(M, g)$ and $(N, h)$ be smooth Riemannian manifolds of dimensions $m$ and $n$, respectively, with $N$ compact without boundary. Using the Nash-Moser Theorem, we embed $N$ isometrically into $\mathbb{R}^{K}$.

We recall that the energy $E(u)$ of a smooth map $u: M \rightarrow N$ is defined as

$$
E(u)=\frac{1}{2} \int_{M}|d u|^{2} d m_{g},
$$

where $d m_{g}$ is the volume form induced by $g$ and $|d u|$ is the Hilbert-Schmidt norm of $d u$ considered as a section of $T M^{*} \otimes u^{*} T N$. A smooth function $u: M \rightarrow N$ is said to be harmonic if it is a critical point of the energy, i.e. if $d^{*} d u=0$ (see [5]). The heat flow for harmonic maps, with initial data $u_{0}: M \rightarrow N$, is the solution to the evolution equation corresponding to the harmonic map problem:

$$
\left(\partial_{t}+d^{*} d\right) u=0, \quad u(0, \cdot)=u_{0}(\cdot) .
$$

Using the embedding $N \rightarrow \mathbb{R}^{K}$ the above equation can be rewritten as

$$
\partial_{t} u+\Delta_{M} u=\operatorname{Tr}_{g} A_{u}(d u, d u), \quad u(0, \cdot)=u_{0}(\cdot),
$$

where $\Delta_{M}$ is the Laplace-Beltrami operator of $(M, g)$; the expression $A_{p}(\cdot, \cdot)$ denotes the second fundamental form at $p \in N$ of the embedding $N \rightarrow \mathbb{R}^{K}$, and $\operatorname{Tr}_{g} A_{u}(d u, d u)$ at $(t, x)$ is the trace of $A_{u(t, x)}(d u(t, x), d u(t, x))$ with respect to $g_{x}$, which is a bilinear form on $T_{x} M$ with values in $\mathbb{R}^{K}$. The advantage of the form (1.1) for the heat flow equation is that it allows us to formulate the problem in a

Received by the editors September 4, 1994.

1991 Mathematics Subject Classification. Primary 35B65, 35D10, 49N60, 35Kxx, 58E20, $58 \mathrm{G} 11$.

Key words and phrases. Heat flow, harmonic maps.

The first author was supported by NSF grant \#DMS-9101911.

(C)1996 American Mathematical Society 
weak sense. Define the space

$$
\begin{aligned}
& H\left(\mathbb{R}^{+} \times M, N\right)=\left\{u \in L^{\infty}\left(\mathbb{R}^{+} \times M, \mathbb{R}^{K}\right): u(t, x) \in N\right. \text { a.e. } \\
& \left.\qquad \partial_{t} u \in L^{2}\left(\mathbb{R}^{+} \times M, \mathbb{R}^{K}\right), d u \in L^{\infty}\left(\mathbb{R}^{+}, L^{2}\left(M, \mathbb{R}^{K}\right)\right)\right\} .
\end{aligned}
$$

Then a weak solution of the heat flow (1.1) is a function $u \in H\left(\mathbb{R}^{+} \times M, N\right)$ that satisfies

$$
\int_{\mathbb{R}^{+} \times M}\left\{\left\langle\partial_{t} u, \eta\right\rangle+\langle d u, d \eta\rangle-\left\langle\eta, \operatorname{Tr}_{g} A(d u, d u)\right\rangle\right\} d m_{g} d t
$$

for any $\eta \in C_{0}^{\infty}\left(\mathbb{R}^{+} \times M, \mathbb{R}^{K}\right)$ as well as the initial condition $u(0, \cdot)=u_{0}(\cdot)$.

The global existence of a weak solution to the problem (1.1) is known; see for example [3]. The weak solution obtained in [3] is smooth outside a set $\Sigma(u) \subseteq$ $\mathbb{R}^{+} \times M$, the singular set, which is closed and has locally finite $m$-dimensional Hausdorff measure (here and hereafter the Hausdorff measure on $\mathbb{R}^{+} \times M$ is defined using the parabolic metric defined in $\S 2$ ). However, Coron showed in [4] that the problem (1.1) may have infinitely many distinct weak solutions. Therefore the partial regularity of weak solutions becomes an interesting problem.

In analogy with the notion of stationary harmonic map (see for example [12], [13]), Feldman introduced in [7] the notion of stationary solution of the heat flow. The stationarity condition can be formulated in the following way.

A vector field $X$ on $\mathbb{R}^{+} \times M$ is future directed if $d t(X) \geq 0$, where $t$ is the first coordinate function on $\mathbb{R}^{+} \times M$.

If $Z=(T, X)$ is a $C^{1}$ vector field of compact support on $\mathbb{R}^{+} \times M$, define $\tilde{u}_{Z, \varepsilon}: \mathbb{R}^{+} \times M \rightarrow N$ as

$$
\tilde{u}_{Z, \varepsilon}(t, x)=u\left(\exp _{t, x}(\varepsilon Z(t, x))\right)=u\left(t+\varepsilon T(t, x), \exp _{x}(\varepsilon X(t, x))\right) .
$$

1.1 Definition ([7]). A weak solution $u$ of the heat flow (1.1) is said to be stationary if for any $C^{1}$ future directed vector field $Z$ on $\mathbb{R}^{+} \times M$ with compact support we have

$$
\int_{\mathbb{R}} \int_{M}\left\langle\partial_{t} u,\left.\frac{\partial}{\partial \varepsilon} u_{Z, \varepsilon}\right|_{\varepsilon=0}\right\rangle d m_{g} d t+\left.\frac{\partial}{\partial \varepsilon}\left(\int_{\mathbb{R}^{+}} E\left(u_{Z, \varepsilon}\right) d t\right)\right|_{\varepsilon=0} \leq 0 .
$$

In [7], Feldman proved that if the target manifold $N$ is the standard $n$-dimensional sphere, then the singular set of a weak stationary solution $u$ of (1.1) has zero $m$-dimensional Hausdorff measure. Independently, Chen, Li and Lin [2] obtained the same result by assuming, instead of stationarity, the monotonicity inequality and energy inequality (see (2.1) and (2.7) in [2]).

Extending previous partial regularity results of Hélein [8], [9] and Evans [6], Bethuel [1] proved that for stationary harmonic maps the singular set has zero $m$-dimensional Hausdorff measure, provided that the solution is stationary.

Thus one would expect that for the heat flow the singular set of a weak stationary solution of the heat flow into arbitrary target manifolds also has zero $m$-dimensional Hausdorff measure. The purpose of this note is a partial result in this direction.

Roughly speaking, we will show that if a weak stationary solution $u$ of the heat flow has small initial energy $E\left(u_{0}\right)$ and if its singular set $\Sigma(u)$ has co-dimension greater than 2 , then $u$ is smooth. More precisely we have

1.2 Theorem. Let $u \in H\left(\mathbb{R}^{+} \times M, N\right)$ be a weak solution of the heat flow (1.1). Assume that:

(1) The weak solution $u$ is stationary. 
(2) The singular set $\Sigma(u)$ of $u$ satisfies the following regularity conditions:

(a) for some strictly positive $\delta_{0}<1$, the set $\Sigma(u)$ is contained in $M \times\left[\delta_{0}^{2}, \infty\right)$;

(b) the set $\Sigma(u)$ has bounded uniform $(m, q)$-density with respect to the parabolic distance for $q>2$.

(3) For some $\varepsilon=\varepsilon\left(m, N, \delta_{0}\right)$ we have $E\left(u_{0}\right)<\varepsilon$.

Then $u$ is smooth in $M$.

For the definition of $(m, q)$-density see Definition 2.3 below. The condition of stationarity can be replaced by assuming the monotonicity inequality (4.5) below and the energy inequality $[2,(2.7)]$. In fact, in [2] it was shown that these inequalities imply the estimates (4.2) and (4.4) below from which Theorem 1.2 follows.

In $\S 3$ we shall prove that the condition of stationarity is automatically satisfied if we assume that the codimension $q$ of the singular set is strictly greater than 2 and that $|d u|$ has higher integrability. See Theorem 3.1 below for a precise statement.

Our assumptions on the singular set are similar to Mou's [11] who proved analogous results for $p$-harmonic maps.

1.3 Notation. Let $\mathcal{L}^{m+1}=d m_{g} d t$. Whenever not explicitly indicated, integration on subsets of $M$ and of $\mathbb{R}^{+} \times M$ will always be intended with respect to the measures $d m_{g}$ and $\mathcal{L}^{m+1}$. By an absolute constant we intend a constant which may depend only on $(M, g)$ and $(N, h)$. Occasionally we shall write $a \ll b$ to mean that for some absolute constant $C$ we have $a<C b$.

1.4 Notation. For $u \in H\left(\mathbb{R}^{+} \times M, N\right)$ we set $u(t)=u(t, \cdot)$.

1.5 Notation. The metric $d t^{2} \otimes g$ induces a connection $\vec{\nabla}$ on $\mathbb{R}^{+} \times M$, for which the hypersurfaces $\{t\} \times M$ are totally geodesics. In particular the pull back of $g$ to $\mathbb{R}^{+} \times M$ (denoted $g$ again) is parallel. We shall denote by $\overrightarrow{d u}$ the differential of $u: \mathbb{R}^{+} \times M \rightarrow N$, while reserving the symbol $d u$ to denote the restriction of $\overrightarrow{d u}$ to $\{t\} \times T M$. Similarly $\vec{\delta} Z$ denotes the divergence $\vec{\delta} Z=-\operatorname{Tr} \vec{\nabla} Z$.

1.6 Remark. The condition of stationarity yields immediately that $u \in$ $H\left(\mathbb{R}^{+} \times M, N\right)$ is a stationary solution of the heat flow iff for any $C^{1}$-future directed vector field $Z$ on $\mathbb{R}^{+} \times M$ with compact support we have:

$$
\int_{\mathbb{R}^{+} \times M}\left\{\left\langle\partial_{t} u, \overrightarrow{d u}(Z)\right\rangle+\langle d u, \overrightarrow{d u}(\vec{\nabla} Z)\rangle+\frac{1}{2}\langle d u, d u\rangle \vec{\delta} Z\right\} \leq 0
$$

where $\langle\cdot, \cdot\rangle$ denotes the inner product on any of the various tensor bundles $T^{(r, s)} M \otimes$ $u^{*} T N$.

1.7 Lemma. If $u \in H\left(\mathbb{R}^{+} \times M, N\right)$ is a weak solution of the heat flow and $u \in$ $H_{\text {loc }}^{2}\left(\Omega_{0}, N\right)$ for some open subset $\Omega_{0} \subseteq \mathbb{R}^{+} \times M$, then $u$ is stationary on $\Omega_{0}$.

Proof (Feldman [7]). A weak solution of the heat flow in $H_{\text {loc }}^{2}\left(\Omega_{0}, N\right)$ is a strong solution of the heat equation (1.1) on $\Omega_{0}$.

Let $Z$ be a vector field of compact support in $\Omega_{0}$. Then, denoting by $\approx$ equality up to divergence terms we have

$$
\begin{aligned}
\frac{1}{2}\|d u\|^{2} \vec{\delta} Z & \approx \frac{1}{2} \vec{\nabla}_{Z}\left(\|d u\|^{2}\right)=\left\langle\vec{\nabla}_{Z} d u, d u\right\rangle \\
& =\langle\nabla(\vec{d} u(Z))-\overrightarrow{d u}(\vec{\nabla} Z), d u\rangle=\left\langle\vec{d} u(Z), \nabla^{*} d u\right\rangle-\langle\vec{d} u(\vec{\nabla} Z), d u\rangle \\
& =-\left\langle\vec{d} u(Z), \partial_{t} u\right\rangle-\langle\vec{d} u(\vec{\Delta} Z), d u\rangle,
\end{aligned}
$$


where in the last line we have used the heat equation (1.1). After integration we obtain the claim.

2.

2.1 Notation. For $z_{1}=\left(t_{1}, x_{1}\right), z_{2}=\left(t_{2}, x_{2}\right)$ in $\mathbb{R}^{+} \times M$, the parabolic distance of $z_{1}$ and $z_{2}$ is defined as $\rho\left(z_{1}, z_{2}\right)=\max \left(\left|t_{1}-t_{2}\right|^{1 / 2},\left|x_{1}-x_{2}\right|\right)$. We set $\rho(z, \Sigma)=$ $\inf \left\{\rho\left(z, z^{\prime}\right) \mid z^{\prime} \in \Sigma\right\}$. For $r>0$, denote

$$
\begin{gathered}
\Sigma_{r}=\left\{z \in \mathbb{R}^{+} \times M \mid \rho(z, \Sigma)<r\right\}, \\
P_{r}(z)=\left\{z^{\prime} \in \mathbb{R} \times M \mid \rho\left(z, z^{\prime}\right)<r\right\} .
\end{gathered}
$$

Recall that $m$ denotes the dimension of $M$.

2.2 Definition. The upper Minkowski $q$-content of a set $\Sigma \in \mathbb{R}^{+} \times M$ with respect to the parabolic metric $\rho$ is the number

$$
\overline{\mathcal{M}}_{\rho}^{q}(\Sigma)=\limsup _{r \rightarrow 0} \frac{\mathcal{L}^{m+1}\left(\Sigma_{r}\right)}{r^{m+2-q}} .
$$

We say that a set $\Sigma \subseteq \mathbb{R}^{+} \times M$ has locally finite upper Minkowski $k$-content, i.e. for any compact set $K$ we have $\overline{\mathcal{M}}_{\rho}^{k}(\Sigma \cap K)<\infty$.

2.3 Definition. We say that a set $\Sigma \subseteq \mathbb{R}^{+} \times M$ has bounded uniform $(m, q)$-density with respect to the parabolic distance if for some $r_{0}>0$ the quantity

$\Phi_{r_{0}}^{(m, q)}(\Sigma)=\sup \left\{\mathcal{L}^{m+1}\left(\Sigma_{r_{1}} \cap P_{r_{2}}(z)\right) /\left(r_{1}^{m+2-q} r_{2}^{q}\right): r_{1}<r_{0}, r_{2}<r_{0}, z \in \mathbb{R}^{+} \times M\right\}$ is finite.

Clearly if $\Sigma \subseteq \mathbb{R}^{+} \times M$ has bounded uniform $(m, q)$-density, then it has locally finite upper Minkowski $q$-content. Both these notions are not vacuous. To see this we give the following definition:

2.4 Definition. A closed set $\Sigma \in \mathbb{R}^{+} \times M$ is rectifiable of parabolic dimension $q$ if, for any compact set $K \subseteq \mathbb{R}^{+} \times M$, there exist finitely many Lipshitzian maps $f_{i}: \mathbb{R}^{q-1} \rightarrow \mathbb{R}^{+} \times M$ and $g_{j}: \mathbb{R}^{q} \rightarrow\left\{t_{j}\right\} \times M$ and compact sets $C_{i} \subseteq \mathbb{R}^{q-1}$ and $D_{j} \subseteq \mathbb{R}^{q}$ such that $\Sigma \cap K \subseteq \bigcup_{i, j} f_{i}\left(C_{i}\right) \cup g_{j}\left(D_{j}\right)$.

Then, it is not difficult to see that we have:

2.5 Proposition. A rectifiable closed set $\Sigma \in \mathbb{R}^{+} \times M$ of parabolic dimension $q$ has locally finite upper Minkowski q-content. Similarly a compact smooth set $\Sigma \in \mathbb{R}^{+} \times M$ of parabolic dimension $q$ has bounded uniform $(m, q)$-density.

3.

The goal of this section is to show that weak solutions of the heat flow are stationary if the singular set is "sufficiently small and regular" and the derivative $d u$ blows-up "slowly" near the singular set.

3.1 Theorem. If $u \in H\left(\mathbb{R}^{+} \times M, N\right) \cap C^{\infty}\left(\mathbb{R}^{+} \times M \backslash \Sigma, N\right)$ is a weak solution of the heat flow, then $u$ is a stationary solution provided that

(a) The singular set $\Sigma$ has locally finite upper Minkowski p-content for some $p<m$.

(b) We have $d u \in L^{q}\left(\mathbb{R}^{+} \times M, T N\right)$ with $q=2(m+2-p) /(m-p)$.

We also reach the same conclusion if 
$\left(\mathrm{a}^{\prime}\right)$ The singular set $\Sigma$ has null upper Minkowski $(m-2)$-content.

$\left(\mathrm{b}^{\prime}\right)$ We have $|d u(z)| \ll 1 / \rho(z, \Sigma)$ for all $z \in \mathbb{R}^{+} \times M \backslash \Sigma$.

We shall make use of the following observation, whose proof is standard.

3.2 Remark. For each compact set $K$ and positive $r$, there exists a smooth cut-off function $\psi_{r}: \mathbb{R}^{+} \times M \rightarrow[0,1]$ with compact support such that $\psi_{r}=0$ on $\Sigma_{r} \cap K$, $\psi_{r}=1$ on $\left(\Sigma_{2 r}\right)^{c} \cap K,\left|\nabla \psi_{r}\right|<2 / r$ and $\left|\partial_{t} \psi_{r}\right|<1 / r^{2}$.

Proof of Theorem 3.1. Set $p_{1}=(m+2-p) /(m-p)$ and $p_{2}=\left(1-1 / p_{1}\right)^{-1}=$ $(m+2-p) / 2$. From (a) we have $\lim _{\sup _{r \rightarrow 0}} \mathcal{L}^{m+1}\left(\Sigma_{r}\right) / r^{m+2-p}<\infty$, which implies $\limsup _{r \rightarrow 0} \mathcal{L}^{m+1}\left(\Sigma_{r}\right)^{1 / p_{2}} / r^{2}<\infty$. Since the measure of $\Sigma_{r}$ goes to zero with $r$, from (b) we have $\lim _{r \rightarrow 0} \int_{\Sigma_{r}}|d u|^{2 p_{1}}=0$ and therefore, applying Hölder's inequality, we obtain

$$
\begin{aligned}
& \limsup _{r \rightarrow 0} \frac{1}{r^{2}} \int_{\Sigma_{r}}|d u|^{2} d \mathcal{L}^{m+1} \\
& \quad \leq \limsup _{r \rightarrow 0} \frac{1}{r^{2}}\left(\int_{\Sigma_{r}}|d u|^{2 p_{1}} d \mathcal{L}^{m+1}\right)^{1 / p_{1}}\left(\mathcal{L}^{m+1}\left(\Sigma_{r}\right)\right)^{1 / p_{2}}=0 .
\end{aligned}
$$

If we assume $\left(a^{\prime}\right)$ and $\left(b^{\prime}\right)$ instead, we obtain

$$
\int_{\Sigma_{r} \backslash \Sigma_{2 r}}|d u|^{2} d \mathcal{L}^{m+1} \ll r^{-2} \mathcal{L}^{m+1}\left(\Sigma_{2 r}\right)
$$

and $\lim _{r \rightarrow 0} \mathcal{L}^{m+1}\left(\Sigma_{2 r}\right) / r^{4}=0$. Thus under both sets of hypotheses we have that

$$
\limsup _{r \rightarrow 0} \frac{1}{r^{2}} \int_{\Sigma_{2 r} \backslash \Sigma_{r}}|d u|^{2} d \mathcal{L}^{m+1}=0 .
$$

Since $\partial_{t} u \in L^{2}\left(\mathbb{R}^{+} \times M, T N\right)$, equation (3.2) implies that

$$
\limsup _{r \rightarrow 0} \frac{1}{r} \int_{\Sigma_{2 r} \backslash \Sigma_{r}}|d u|\left|\partial_{t} u\right| d \mathcal{L}^{m+1}=0 .
$$

Now, let $Z$ be a $C^{1}$ future-oriented vector field of compact support $K$ on $\mathbb{R}^{+} \times M$. Fix $r>0$ and let $\psi_{r}: \mathbb{R}^{+} \times M \rightarrow[0,1]$ be a smooth cut-off function as in Remark 3.2. Then $\psi_{r} Z$ is also future-oriented and $C^{1}$, and supported in a region on which $u$ is smooth. Assuming that $K \subseteq\left[t_{1}, t_{2}\right] \times M$, by Lemma 1.7, we have

$$
\int_{t_{1}}^{t_{2}} \int_{M}\left\{\left\langle\partial_{t} u, \vec{d} u\left(\psi_{r} Z\right)\right\rangle+\left\langle d u, \overrightarrow{d u}\left(\vec{\nabla} \psi_{r} Z\right)\right\rangle+\frac{1}{2}\langle d u, d u\rangle \vec{\delta}\left(\psi_{r} Z\right)\right\} \leq 0
$$

from which we derive

$$
\begin{array}{r}
\int_{t_{1}}^{t_{2}} \int_{M} \psi_{r}\left\{\left\langle\partial_{t} u, \overrightarrow{d u}(Z)\right\rangle+\langle d u, \overrightarrow{d u}(\vec{\nabla} Z)\rangle+\frac{1}{2}\langle d u, d u\rangle \vec{\delta} Z\right\} \\
\leq \int_{\Sigma_{2 r} \backslash \Sigma_{r}}\left\{\left\langle d u, \overrightarrow{d u}(Z) \otimes d \psi_{r}\right\rangle-\frac{1}{2}\langle d u, d u\rangle \vec{d} \psi_{r}(Z)\right\} .
\end{array}
$$

As we let $r$ tend to 0 the left-hand side of (3.5) converges to

$$
\int_{t_{1}}^{t_{2}} \int_{M}\left\{\left\langle\partial_{t} u, \overrightarrow{d u}(Z)\right\rangle+\langle d u, \overrightarrow{d u}(\vec{\nabla} Z)\rangle+\frac{1}{2}\langle d u, d u\rangle \vec{\delta} Z\right\} .
$$

Thus, in order to establish the proposition, it is sufficient to show that the righthand side of (3.5) has a non-positive upper limit. 
Writing $Z=T \partial_{t}+X$ with $d t(X)=0$, the right-hand side of (3.5) is equal to

$$
\begin{aligned}
\int_{\Sigma_{2 r} \backslash \Sigma_{r}}\left\{T\left\langle d u, \partial_{t} u \otimes d \psi_{r}\right\rangle\right. & +\left\langle d u, d u(X) \otimes d \psi_{r}\right\rangle \\
& \left.-\frac{1}{2}\langle d u, d u\rangle\left(T \partial_{t} \psi_{r}+d \psi_{r}(X)\right)\right\},
\end{aligned}
$$

and, using $\left|d \psi_{r}\right|<2 / r$ and $\left|\partial_{t} \psi_{r}\right|<1 / r^{2}$, we obtain that the expression (3.6) is dominated by

$$
\begin{gathered}
\int_{\Sigma_{2 r} \backslash \Sigma_{r}}\left\{\frac{2}{r}|T||d u|\left|\partial_{t} u\right|+\frac{2}{r}|X||d u|^{2}+\frac{1}{2} \frac{1}{r^{2}}|T||d u|^{2}+\frac{1}{2} \frac{2}{r}|X||d u|^{2}\right\} d \mathcal{L}^{m+1} \\
<2\|Z\|_{\infty} \int_{\Sigma_{2 r} \backslash \Sigma_{r}}\left\{\frac{1}{r^{2}}|d u|^{2}+\frac{1}{r}|d u|\left|\partial_{t} u\right|\right\} d \mathcal{L}^{m+1}
\end{gathered}
$$

which by (3.2)-(3.3) goes to zero as $r \rightarrow 0$.

4.

Henceforth we assume, for simplicity, that $M=\mathbb{R}^{m}$ with the ordinary Euclidean metric.

4.1 Lemma. If $u \in H\left(\mathbb{R}^{+} \times M, N\right)$ is a weak stationary solution of the heat flow, then

(1) The energy is decreasing at almost all times:

$$
E(u(t))<E(u(0)) \text { for a.e. } t \in \mathbb{R}^{+} .
$$

(2) There is an absolute constant $C_{1}$ such that:

$$
r^{2-m} \int_{P_{r / 2}(z)}\left|\partial_{t} u\right|^{2}<C_{1} r^{-m} \int_{P_{r}(z)}|d u|^{2}
$$

for any ball $P_{r}(z) \subset \mathbb{R}^{+} \times M$.

Proof. These are Propositions 10-12 in [7].

4.2 Notation. For $u \in H\left(\mathbb{R}^{+} \times M, N\right)$ and any ball $P_{r}(z) \subseteq \mathbb{R}^{+} \times M$ we define the normalized energy in the r-ball about $z$ as

$$
N_{r}(u, z)=N\left(u, P_{r}(z)\right)=r^{-m} \int_{P_{r}(z)}|d u|^{2} .
$$

4.3 Lemma (Monotonicity property). There is an absolute constant $C_{2}$ such that, for any weak stationary solution of the heat flow $u \in H\left(\mathbb{R}^{+} \times M, N\right)$ and for any ball $P_{r}(z) \subseteq \mathbb{R}^{+} \times M$, we have:

$$
N_{r^{\prime}}\left(u, z^{\prime}\right)<C_{2} N_{r}(u, z) \quad \text { for all } z^{\prime} \in P_{r / 4}(z) \text { and } r^{\prime}<r / 8 \text {. }
$$

Proof. Let $\beta \in(0,1)$ be a fixed constant and let $\phi: \mathbb{R}^{m} \rightarrow[0,1]$ be a $C^{\infty}$ function with support in $\left(B_{1 / 4}(0)\right)$ and with $\phi \equiv 1$ on $B_{1 / 8}(0)$. For any $z_{0} \in \mathbb{R}^{+} \times M$, and any $R \in\left(0, \frac{\sqrt{t_{0}}}{2 \beta}\right)$, define

$$
\Phi_{\beta}\left(R, u, z_{0}\right)=\frac{\beta^{2} R^{2}}{2} \int_{\left\{t_{0}-\beta^{2} R^{2}\right\} \times M}|d u|^{2} G_{z_{0}} \phi_{\beta}^{2} d m_{g},
$$


and

$$
\Psi_{\beta}\left(R, u, z_{0}\right)=\frac{1}{2} \int_{t_{0}-4 \beta^{2} R^{2}}^{t_{0}-\beta^{2} R^{2}} \int_{M}|d u|^{2} G_{z_{0}} \phi_{\beta}^{2} d \mathcal{L}^{m+1},
$$

where $G_{z_{0}}:\left(-\infty, t_{0}\right] \times M \rightarrow R^{+}$is the solution of the equation $\left(\partial_{t}+\Delta\right) G_{z_{0}}=0$ of the backward heat flow on $M$ which satisfies the final condition $G_{z_{0}}\left(t_{0}\right)=\delta_{x_{0}}$, and where we have set $\phi_{\beta}(x)=\phi\left(\frac{x-x_{0}}{\beta}\right)$.

We claim that there exists an absolute constant $C>0$ such that for any $z_{0} \in$ $M \times R_{+}$, and $0<R_{1} \leq R_{2} \leq \min \left(\frac{\sqrt{t_{0}}}{2 \beta}, 1 / 4\right)$, we have

$$
\Psi_{\beta}\left(R_{1}, u, z_{0}\right) \leq \Psi_{\beta}\left(R_{2}, u, z_{0}\right)+C \beta^{-m} \int_{P_{\beta / 2}\left(z_{0}\right)}|d u|^{2} d \mathcal{L}^{m+1} .
$$

Since the equation (1.1) is invariant under the translation $z \rightarrow z-z_{0}$, we may assume, to simplify the notation, that $z_{0}=(0,0)$. We denote $G=G_{(0,0)}, P_{r}=$ $P_{r}(0,0)$ and $B_{r}=B_{r}(0)$.

By the argument in [7] (see the derivation of $(24)$ and (25) in [7]), it is not difficult to obtain the following estimates (take $\theta=t G \phi_{\beta}^{2}, \zeta=x G \phi_{\beta}^{2}$ ):

$$
\begin{aligned}
& \int_{\left\{t_{2}\right\} \times M}-\int_{\left\{t_{1}\right\} \times M} t|d u|^{2} G \phi_{\beta}^{2} d m_{g} \\
& \geq \int_{t_{1}}^{t_{2}} \int_{M}\left[-|d u|^{2}\right.\left(\frac{n-2}{2}+\frac{|x|^{2}}{4 t}\right) \\
&\left.-\left\langle\partial_{t} u \otimes x, d u\right\rangle-2\left|\partial_{t} u\right|^{2} t\right] G \phi_{\beta}^{2} d \mathcal{L}^{m+1}+D_{1}
\end{aligned}
$$

and

$$
\int_{t_{1}}^{t_{2}} \int_{M}\left[\left\langle\partial_{t} u \otimes x, d u\right\rangle-|d u|^{2}\left(\frac{n-2}{2}+\frac{|x|^{2}}{4 t}\right)+\frac{|d u \cdot x|^{2}}{2 t}\right] G \phi_{\beta}^{2} d \mathcal{L}^{m+1}+D_{2}=0,
$$

where

$$
\begin{aligned}
D_{1} & =\int_{t_{1}}^{t_{2}} \int_{M} \frac{4 t}{\beta}\left\langle\partial_{t} u \otimes d \phi(x / \beta), d u\right\rangle \phi_{\beta} G d \mathcal{L}^{m+1} \\
& =\int_{t_{1}}^{t_{2}} \int_{M} \frac{4 t}{\beta} \partial_{t} u^{\alpha} \partial_{j} \phi(x / \beta) \partial_{j} u^{\alpha} \phi_{\beta} G d \mathcal{L}^{m+1}
\end{aligned}
$$

and

$$
D_{2}=\int_{t_{1}}^{t_{2}} \int_{M}\left[-\frac{1}{\beta}|d u|^{2} x^{j} \partial_{j} \phi(x / \beta) \phi_{\beta} G+\frac{2}{\beta} \partial_{j} u^{\alpha} \partial_{k} u^{\alpha} x^{k} \phi_{\beta} \partial_{j} \phi(x / \beta) G\right] d \mathcal{L}^{m+1} .
$$

Let $t_{1}=-R_{2}^{2} \beta^{2}, t_{2}=-R_{1}^{2} \beta^{2}$; we have

$$
\begin{aligned}
\left|D_{1}\right| \leq & \int_{-R_{2}^{2} \beta^{2}}^{-R_{1}^{2} \beta^{2}} \int_{M} \beta^{2-m}|t|^{m / 2}\left|\partial_{t} u\right|^{2} G \phi_{\beta}^{2} d \mathcal{L}^{m+1} \\
& +C \int_{-R_{2}^{2} \beta^{2}}^{-R_{1}^{2} \beta^{2}} \int_{M} \beta^{m-4}|t|^{(4-m) / 2}|d u|^{2} G|d \phi|^{2}\left(\frac{x}{\beta}\right) d \mathcal{L}^{m+1} \\
= & D_{11}+D_{12}
\end{aligned}
$$


By Lemma 4.1,

$$
\begin{aligned}
\left|D_{11}\right| & \leq C \int_{-R_{2}^{2} \beta^{2}}^{-R_{1}^{2} \beta^{2}} \int_{B_{\beta / 4}} \beta^{2-m}\left|\partial_{t} u\right|^{2} d \mathcal{L}^{m+1} \\
& \leq C \int_{P_{\beta / 4}} \beta^{2-m}\left|\partial_{t} u\right|^{2} d \mathcal{L}^{m+1} \leq C \beta^{-m} \int_{P_{\beta / 2}}|d u|^{2} d \mathcal{L}^{m+1}
\end{aligned}
$$

Noticing that on $\{\beta / 8 \leq|x| \leq \beta / 4\} \times\left\{-R_{2}^{2} \beta^{2} \leq t \leq-R_{1}^{2} \beta^{2}\right\}$,

$$
|t|^{(4-m) / 2} G \leq C|t|^{2-m} \exp \left\{-\frac{\beta^{2}}{256|t|}\right\} \leq C \beta^{4-2 m},
$$

we obtain

$$
\left|D_{12}\right| \leq C \beta^{-m} \int_{P_{\beta / 2}}|d u|^{2} d \mathcal{L}^{m+1}
$$

Formulas (4.8) and (4.9) lead to

$$
\left|D_{1}\right| \leq C \beta^{-m} \int_{P_{\beta / 2}}|d u|^{2} d \mathcal{L}^{m+1}
$$

Similarly, we obtain

$$
\left|D_{2}\right| \leq C \beta^{-m} \int_{P_{\beta / 2}}|d u|^{2} d \mathcal{L}^{m+1} .
$$

Subtracting (4.7) from (4.6), and taking $t_{1}=-R_{2}^{2} \beta^{2}, t_{2}=-R_{1}^{2} \beta^{2}$, we obtain

$$
\begin{aligned}
& \Phi_{\beta}\left(R_{2}, u, 0\right)-\Phi_{\beta}\left(R_{1}, u, 0\right) \\
& \quad \geq \int_{-R_{2}^{2} \beta^{2}}^{-R_{1}^{2} \beta^{2}} \int_{M}-\left[2 \partial_{t} u d u \cdot x+\frac{|d u \cdot x|^{2}}{2 t}+2\left|\partial_{t} u\right|^{2} t\right] G \phi_{\beta}^{2} d \mathcal{L}^{m+1}+D_{1}-D_{2} .
\end{aligned}
$$

From (4.10)-(4.11) and the inequality

$$
\left|2\left\langle\partial_{t} u \otimes x, d u\right\rangle\right| \leq \frac{\left|\partial_{j} u x^{j}\right|^{2}}{2 t}+2|t|\left|\partial_{t} u\right|^{2},
$$

we see that (4.12) yields

$$
\Phi_{\beta}\left(R_{1}, u, 0\right) \leq \Phi_{\beta}\left(R_{2}, u, 0\right)+C \beta^{-m} \int_{P_{\beta / 2}}|d u|^{2} d \mathcal{L}^{m+1} .
$$

Observing that

$$
\Psi_{\beta}(R, u, 0)=\frac{2}{R} \int_{R}^{2 R} \Phi_{\beta}(r, u, 0) d r,
$$

we conclude from (4.13) that we have

$$
\Psi_{\beta}\left(R_{1}, u, 0\right) \leq \Psi_{\beta}\left(R_{2}, u, 0\right)+C \beta^{-m} \int_{P_{\beta / 2}}|d u|^{2} d \mathcal{L}^{m+1} .
$$

Thus the claim (4.5) is proved. 
As in the proof of Lemma 2.2 in [2], we have for any $0<r^{\prime} \leq r / 8$

$$
\begin{aligned}
& r^{\prime-m} \int_{P_{r^{\prime}}\left(z_{0}\right)}|d u|^{2} d \mathcal{L}^{m+1} \leq C \Psi_{r}\left(r^{\prime} / r, u,\left(x_{0}, t_{0}+2 r^{\prime 2}\right)\right) \\
& \quad \leq C \Psi_{r}\left(1 / 4, u,\left(x_{0}, t_{0}+2 r^{\prime 2}\right)\right)+C r^{-m} \int_{P_{r / 2}\left(x_{0}, t_{0}+2 r^{\prime 2}\right)}|d u|^{2} d \mathcal{L}^{m+1} \\
& \quad \leq C r^{-m} \int_{P_{r / 2}\left(x_{0}, t_{0}+2 r^{\prime 2}\right)}|d u|^{2} d \mathcal{L}^{m+1} \leq C r^{-m} \int_{P_{r}\left(z_{0}\right)}|d u|^{2} d \mathcal{L}^{m+1} .
\end{aligned}
$$

To prove Theorem 1.2 we will require two lemmata. The first lemma states that in a region where $u$ is smooth we can estimate $|d u|^{2}$ by its average.

4.4 Lemma. Let $u \in H\left(\mathbb{R}^{+} \times M, N\right)$ be a weak stationary solution of the heat flow. There are absolute constants $\varepsilon_{1}$ and $C_{3}$ such that if $u$ is smooth on $P_{r}\left(z_{0}\right) \subseteq \mathbb{R}^{+} \times M$ and $N_{r}\left(u, z_{0}\right)<\varepsilon_{1}$, then

$$
\sup _{P_{r / 16}\left(z_{0}\right)}|d u|^{2}<C_{3} r^{-m-2} \int_{P_{r}(z)}|d u|^{2} .
$$

Proof. We follow Schoen and Uhlenbeck [12, Theorem 2.2]. Bochner's formula for $\Delta|d u|^{2}$ (see [5]) implies the estimate

$$
\left(\partial_{t}-\Delta\right)|d u|^{2} \leq C_{N}|d u|^{4}
$$

where $C_{N}$ is the norm of the Riemannian curvature of $(N, h)$.

Let $z^{\prime} \in P_{r}\left(z_{0}\right)$ and $P_{\alpha}\left(z^{\prime}\right) \subseteq P_{r}\left(z_{0}\right)$. If on $P_{\alpha}\left(z^{\prime}\right)$ we have $|d u|^{2}<B$, then

$$
\left(\partial_{t}-\Delta\right)|d u|^{2} \leq C_{N} B|d u|^{2}, \quad \text { on } P_{\alpha}\left(z^{\prime}\right),
$$

and, applying Moser's mean value inequality [10, Formula (3.12)] for parabolic equations in the rectangle $P_{\alpha}\left(z^{\prime}\right)$, we obtain

$$
|d u|^{2}\left(z^{\prime}\right)<\gamma e^{C_{N} B \alpha^{2}} \alpha^{-2} N_{\alpha}\left(u, z^{\prime}\right),
$$

where the constant $\gamma$ depends only on $m$.

Choose $\tau_{0} \in(0, r / 8)$ so that

$$
\left(r / 8-\tau_{0}\right)^{2} \sup _{P_{\tau_{0}}\left(z_{0}\right)}|d u|^{2}=\max _{0 \leq \tau \leq r / 8}\left[(r / 8-\tau)^{2} \sup _{P_{\tau}\left(z_{0}\right)}|d u|^{2}\right]
$$

and pick $\bar{z} \in P_{\tau_{0}}\left(z_{0}\right)$ so that

$$
|d u|^{2}(\bar{z})=\max _{P_{\tau_{0}}\left(z_{0}\right)}|d u|^{2}=e_{0} .
$$

Set $\rho_{0}=\frac{1}{2}\left(r / 8-\tau_{0}\right)$. Then $\tau_{0}+\rho_{0} \leq r / 8$ and therefore

$$
\max _{P_{\rho_{0}}(\bar{z})}|d u|^{2} \leq \max _{P_{\rho_{0}+\tau_{0}}(z)}|d u|^{2} \leq \frac{\left(r / 8-\tau_{0}\right)^{2}}{\left(r / 8-\rho_{0}-\tau_{0}\right)^{2}}|d u|^{2}(\bar{z})=4 e_{0} .
$$

If $\rho_{0}>\left(e_{0} C_{N}\right)^{-1 / 2}$, then applying (4.15) with $z^{\prime}=\bar{z}$ and $\alpha=\left(e_{0} C_{N}\right)^{-1 / 2}$ we obtain

$$
e_{0}=|d u|^{2}(\bar{z})<\gamma e^{4} e_{0} C_{N} N_{\left(e_{0} C_{N}\right)^{-1 / 2}}(u, \bar{z})<\gamma e^{4} e_{0} C_{N} C_{1} N_{r}\left(u, z_{0}\right) .
$$

In the last step, we have used the monotonicity inequality and the fact that $\left(e_{0} C_{N}\right)^{-1 / 2}<\rho_{0}<r / 16$ and $\bar{z} \in P_{r / 8}\left(z_{0}\right)$. The last inequality is a contradiction 
if $N_{r}\left(u, z_{0}\right)$ is smaller than $\varepsilon_{1}=\gamma^{-1} e^{-4} C_{N}^{-1}$. Thus we can assume that $\rho_{0}<$ $\left(e_{0} C_{N}\right)^{-1 / 2}$. Applying (4.15) with $z^{\prime}=\bar{z}$ and $\alpha=\rho_{0}$ we obtain

$$
e_{0}=|d u|^{2}(\bar{z})<\gamma e^{4} \rho^{-2} N_{\rho_{0}}(u, \bar{z})<\gamma e^{4} \rho^{-2} C_{1} N_{r}\left(u, z_{0}\right)
$$

and therefore

$$
(r / 8-\tau)^{2} \sup _{P_{\tau}\left(z_{0}\right)}|d u|^{2} \leq 4 \rho^{2} e_{0} \leq \gamma e^{4} C_{1} N_{r}\left(u, z_{0}\right) .
$$

Choosing $\tau=r / 16$ and $C_{3}=64 \gamma e^{4} C_{1}$ we conclude that:

$$
\sup _{P_{\tau}\left(z_{0}\right)}|d u|^{2} \leq C_{3} r^{-m-2} \int_{P_{r}(z)}|d u|^{2} .
$$

4.5 Lemma. Assume that $u \in H\left(\mathbb{R}^{+} \times M, N\right)$ is a weak stationary solution of the heat flow with singular set $\Sigma \subseteq \mathbb{R}^{+} \times M$ satisfying the condition (2) of Theorem 1.2. There exist constants $\sigma=\sigma\left(m, N, \delta_{0}\right) \in(0,1 / 4)$ and $\varepsilon_{1}=\varepsilon_{1}\left(m, N, \delta_{0}\right)$ such that for any $z \in M \times\left[\delta_{0}^{2}, \infty\right)$ and $r<\min \left(1 / 4, \delta_{0} / 4\right)$ the inequality $N_{r}(u, z)<\varepsilon_{1}$ implies

$$
N_{\sigma r}(u, z)<\frac{1}{2} N_{r}(u, z) .
$$

Proof. Arguing by contradiction, we fix sequences $\varepsilon_{i}$ and $\sigma_{i}<1 / 4$ decreasing to zero and we assume that there exist sequences of points $z_{i} \in\left[\delta_{0}^{2}, \infty\right) \times M$ and $r_{i}<\min \left(1 / 4, \delta_{0} / 4\right)$ such that

$$
\lambda_{i}^{2}:=\frac{1}{2} N_{r_{i}}\left(u, z_{i}\right)<\varepsilon_{i} \text { for all } i
$$

and

$$
N_{\sigma_{i} r_{i}}\left(u, z_{i}\right) \geq \frac{1}{2} N_{r_{i}}\left(u, z_{i}\right) \quad \text { for all } i .
$$

Define

$$
a_{i}=\frac{1}{\operatorname{Vol}\left(P_{r / 2}\left(z_{i}\right)\right)} \int_{P_{r / 2}\left(z_{i}\right)} u
$$

and set

$$
v_{i}(t, x)=\lambda_{i}^{-1} u\left(r_{i}^{2} t+t_{i}, r_{i} x+x_{i}\right) .
$$

Then $v_{i}: P_{1} \subseteq \mathbb{R} \times M \rightarrow N$ is a stationary solution of the heat flow satisfying

$$
N_{1}\left(v_{i}, 0\right)=\lambda_{i}^{-2} N_{r_{i}}\left(u, z_{i}\right)=1
$$

and

$$
N_{\sigma_{i}}\left(v_{i}, 0\right)=\lambda_{i}^{-2} N_{\sigma_{i} r_{i}}\left(u, z_{i}\right) \geq \frac{\lambda_{i}^{-2}}{2} N_{r_{i}}\left(u, z_{i}\right)=\frac{1}{2} .
$$

From (4.2) and (4.23) we obtain

$$
\int_{P_{1 / 2}}\left|\partial_{t} v_{i}\right|^{2}<C_{1}
$$

and therefore, using the Poincaré inequality,

$$
\int_{P_{1 / 2}}\left|v_{i}\right|^{2}<C_{2} .
$$


We can assume then, by possibly passing to a subsequence, that as $i \rightarrow \infty$ we have

$$
\begin{gathered}
v_{i} \rightarrow \bar{v} \text { strongly in } L^{2}\left(P_{1 / 2}, \mathbb{R}^{K}\right), \\
d v_{i} \rightarrow d \bar{v} \text { weakly in } L^{2}\left(P_{1}, \mathbb{R}^{K}\right), \\
\partial_{t} v_{i} \rightarrow \partial_{t} \bar{v} \text { weakly in } L^{2}\left(P_{1 / 2}, \mathbb{R}^{K}\right) .
\end{gathered}
$$

Choose any smooth map $\omega: \mathbb{R} \times M \rightarrow R^{K}$ with support in $P_{1 / 2}$. Then since $v_{i}$ are weak solutions of the heat equation (1.1) we have

$$
\int_{\mathbb{R} \times M}\left\langle\partial_{t} v_{i}, \omega\right\rangle+\left\langle d v_{i}, d \omega\right\rangle=\lambda_{i} \int_{\mathbb{R} \times M} A\left(a_{i}+\lambda_{i} v_{i}\right)\left(d v_{i}, d v_{i}\right) \omega .
$$

By the compactness of $N$ the right-hand side is bounded by $C_{3} \lambda_{i} N_{1 / 2}\left(v_{i}\right)\|\omega\|_{\infty}$, and therefore as $i \rightarrow \infty$ we obtain from (4.26), (4.27)

$$
\int_{\mathbb{R} \times M}\left\langle\partial_{t} \bar{v}, \omega\right\rangle+\langle d \bar{v}, d \omega\rangle=0
$$

Hence the limit $\bar{v}$ solves the heat equation $\left(\partial_{t}+\Delta\right) \bar{v}=0$ weakly and also classically. From the local estimate of the parabolic equations and (4.23), (4.26) we obtain that

$$
\|d \bar{v}\|_{L^{\infty}\left(P_{1 / 4}\right)} \leq C_{4} N_{1 / 2}(\bar{v}) .
$$

\subsection{Claim.}

$$
d v_{i} \rightarrow d \bar{v} \text { strongly in } L^{2}\left(P_{1 / 4}, \mathbb{R}^{K}\right) .
$$

Fix $\sigma$ so that $N_{\sigma}(\bar{v})<1 / 4$. From Claim 4.6 and (4.24) we obtain

$$
\frac{1}{4}>N_{\sigma}(\bar{v})=\lim _{i} N_{\sigma}\left(v_{i}\right) \geq \lim _{i} N_{\sigma_{i}}\left(v_{i}\right) \geq \frac{1}{2},
$$

which is a contradiction. Thus in order to conclude our proof, it suffices to prove Claim 4.6.

Proof of Claim 4.6. Recall that $\Sigma(u)$ denotes the singular set of $u$. For simplicity we define for $z=(t, x) \in P_{1}$

$$
\phi_{i}(t, x)=\left(r_{i}^{2} t+t_{i}, r_{i} x+x_{i}\right) .
$$

Thus $v_{i}=\lambda_{i}^{-1} u \circ \phi_{i}$ on $P_{1}$.

Given an integer $\nu=4^{l}$ with $l>3$, let $\mathcal{P}_{\nu}$ be the partition $P_{1}$ into the parallelepipeds with vertices on the lattice $\left\{\left(2 \nu^{-2} j_{0}, 2 \nu^{-1} j_{1}, \ldots, 2 \nu^{-1} j_{m}\right):\left(j_{0}, j_{1}, \ldots, j_{m}\right)\right.$ $\left.\in \mathbb{Z}^{m}\right\}$. We say that a parallelepiped $P \in P_{\nu}$ is $v_{i}$-good if the function $v_{i}$ is smooth on $P$ and on all parallelepipeds contiguous to $P$.

By passing to a subsequence of $v_{i}$, still denoted by $v_{i}$, we have that each $P$ is either $v_{i}$-good for all $i$ or $v_{i}$-bad for all $i$. Thus we can simply say that $P \in \mathcal{P}$ is good or bad.

Denote by $\mathcal{G}_{\nu}$ the collection of good parallelepipeds meeting $P_{1 / 4}$ and let $G_{\nu}=$ $\bigcup_{P \in \mathcal{G}_{\nu}} P$ be their union. Let us also denote the collection of bad parallelepipeds meeting $P_{1 / 4}$ by $\mathcal{B}_{\nu}$ and their union by $B_{\nu}$.

Let $P \in \mathcal{G}_{\nu}$. Clearly any point in $P$ has parabolic distance greater than $\nu^{-1}$ from the singular set $\Sigma_{i}=\phi_{i}^{-1}(\Sigma(u))$ of $v_{i}$. This implies that $\phi_{i}(P)$ has parabolic distance greater than $r_{i} \nu^{-1}$ from $\Sigma(u)$. Therefore we obtain that for $z \in P$,

$$
\left|d v_{i}\right|^{2}(z)=\lambda_{i}^{-2} r_{i}^{2}|d u|^{2}\left(\phi_{i}(z)\right) .
$$


By the monotonicity formula (4.4), we have

$$
N_{16 r_{i} \nu^{-1}}\left(u, \phi_{i}(z)\right)<C_{2} N_{r_{i} / 8}\left(u, \phi_{i}(z)\right) \leq C_{2} N_{r_{i}}\left(u, z_{i}\right)=C_{2} \lambda_{i}^{2},
$$

and therefore for large $i$ we have $N_{16 r_{i} \nu^{-1}}\left(u, \phi_{i}(z)\right)<\varepsilon_{0}$, where $\varepsilon_{0}$ is the constant appearing in Lemma 4.4. Applying now this lemma we obtain that

$$
\begin{aligned}
|d u|^{2}\left(\phi_{i}(z)\right) & <C_{3}\left(r_{i} \nu^{-1}\right)^{-2} N_{r_{i} \nu^{-1}}\left(u, \phi_{i}(z)\right) \\
& <C_{2} C_{3}\left(r_{i} \nu^{-1}\right)^{-2} N_{16 r_{i} \nu^{-1}}\left(u, \phi_{i}(z)\right) \\
& <C_{2} C_{3}\left(r_{i} \nu^{-1}\right)^{-2} \lambda_{i}^{2} .
\end{aligned}
$$

Therefore (4.28) and (4.29) imply that for $z \in P$ and $P \in \mathcal{G}_{\nu}$

$$
\left|d v_{i}\right|^{2}(z)<C_{2} C_{3} \nu^{2}
$$

and by passing to a subsequence we obtain that $d v_{i}$ converges strongly in $L^{2}$ on $G_{\nu}$.

Now we estimate $\int_{B_{\nu}}\left|d v_{i}\right|^{2}$. Observe that if $z \in P_{1 / 4}$ belongs to a bad parallelepiped $P$, then there is a point of $\Sigma_{i}$ in $P$ or in a parallelepiped contiguous to $P$. Then we have $\rho\left(z, \Sigma_{i}\right)<2 \nu^{-1}$ and therefore $\rho\left(\phi_{i}(z), \Sigma(u)\right)<2 r_{i} \nu^{-1}$. Then

$$
\mathcal{L}^{m+1}\left(B_{\nu}\right)<\mathcal{L}^{m+1}\left(P_{1 / 4} \cap\left(\Sigma_{i}\right)_{2 \nu^{-1}}\right)<r_{i}^{-m-2} \mathcal{L}^{m+1}\left(P_{r_{i} / 4}\left(z_{i}\right) \cap \Sigma(u)_{2 r_{i} \nu^{-1}}\right) .
$$

By our assumption on $\Sigma(u)$ we have that

$$
\mathcal{L}^{m+1}\left(P_{r_{i} / 4}\left(z_{i}\right) \cap \Sigma(u)_{2 r_{i} \nu^{-1}}\right)<C\left(r_{i} / 4\right)^{m+2-q}\left(2 r_{i} \nu^{-1}\right)^{q}=C^{\prime} r_{i}^{m+2} \nu^{-q}
$$

and we conclude that

$$
\mathcal{L}^{m+1}\left(B_{\nu}\right)<C^{\prime} \nu^{-q} .
$$

Since each parallelepiped in $\mathcal{P}$ has $\mathcal{L}$-measure equal to $2^{m+1} \nu^{-m-2}$, we obtain that $\mathcal{B}_{\nu}$ has at most $C^{\prime} \nu^{m+2-q}$ elements. Thus, denoting by $c(P, i)$ the centre of $\phi_{i}(P)$ we have

$$
\begin{aligned}
\int_{B_{\nu}}\left|d v_{i}\right|^{2} & =\sum_{P \in \mathcal{B}_{\nu}} \int_{P}\left|d v_{i}\right|^{2}=\sum_{P \in \mathcal{B}_{\nu}}\left(\mathcal{L}^{m+1}(P)\right)^{m /(m+2)} N\left(v_{i}, P\right) \\
& =2^{m(m+1) /(m+2)} \nu^{-m} \sum_{P \in \mathcal{B}_{\nu}} N\left(v_{i}, P\right) \\
& =2^{m(m+1) /(m+2)} \nu^{-m} \sum_{P \in \mathcal{B}_{\nu}} \lambda_{i}^{-2} N\left(u, \phi_{i}(P)\right) \\
& =2^{m(m+1) /(m+2)} \nu^{-m} \sum_{P \in \mathcal{B}_{\nu}} \lambda_{i}^{-2} N_{r^{i} \nu^{-1}}(u, c(P, i)) \\
& <2^{m} \nu^{-m} \sum_{P \in \mathcal{B}_{\nu}} C 2^{m} \lambda_{i}^{-2} N_{r^{i}}\left(u, z_{i}\right) \\
& =C 2^{2 m} \nu^{-m} \sum_{P \in \mathcal{B}_{\nu}} 1 \\
& <C 2^{2 m} \nu^{-m} C^{\prime} \nu^{m+2-q}=2^{2 m} C C^{\prime} \nu^{2-q}=C^{\prime \prime \prime} \nu^{2-q}
\end{aligned}
$$

We conclude that

$$
\limsup _{i} \int_{P_{1 / 4}}\left|d v_{i}\right|^{2} \leq \int_{G_{\nu}}|d \bar{v}|^{2}+C^{\prime \prime \prime} \nu^{2-q}
$$


Since $q>2$, letting $\nu \rightarrow \infty$ we obtain that

$$
\limsup _{i} \int_{P_{1 / 4}}\left|d v_{i}\right|^{2} \leq \int_{G_{\nu}}|d \bar{v}|^{2} .
$$

This implies our claim.

Proof of Theorem 1.2. Since $u$ is a weak stationary solution of the heat equation (1.1), from the monotonicity inequality (4.4) and the energy inequality (4.1) we have

$$
N_{r}(u, z)<C_{2} N_{r_{0}}(u, z)<C_{2} r_{0}^{2-m} E\left(u_{0}\right)
$$

for any $z=(x, t) \in M \times\left[\delta_{0}^{2}, \infty\right)$ and any $r<r_{0} / 8=\delta_{0} / 8$.

Let $\varepsilon_{1}$ be determined as in Lemma 4.5. Then, if $E\left(u_{0}\right)$ is smaller than $\varepsilon_{1} C_{2}^{-1} r_{0}^{m-2}$, we obtain that $N_{r}(u, z)<\varepsilon_{1}$ for any $z=(x, t) \in M \times\left[\delta_{0}^{2}, \infty\right)$ and any $r<\delta_{0} / 8$. Using Lemma 4.5 and a standard iteration argument (see [2, Lemma 5.1], for example), we obtain the Morrey type estimate

$$
\int_{P_{r}(z)}\left(|d u|^{2}+r^{2}\left|\partial_{t} u\right|^{2}\right) \ll r^{m+\alpha}
$$

which implies that $u$ is $C^{\alpha / 2}$ in some ball around $z$. The fact that $u$ is $C^{\infty}$ follows as in [7, Lemma 21].

\section{REFERENCES}

1. F. Bethuel On the singular set of stationary harmonic maps, Manuscripta Math. 78 (1993), 417-443. MR 94a:58047

2. Y. Chen, J. Li, and F. H. Lin Partial regularity for weak heat flows into spheres, Comm. Pure Appl. Math. (to appear).

3. Y. Chen and M. Struwe Existence and partial regularity for heat flow for harmonic maps, Math. Z. 201 (1989), 83-103. MR 90i:58031

4. J. M. Coron Nonuniqueness for the heat flow of harmonic maps, Ann. Inst. H. Poincaré Anal. Non Linéaire 7 (1990), 335-344. MR 91g:58058

5. J. Eells and J. Sampson Harmonic mappings of Riemannian manifolds, Amer. J. Math. 86 (1964), 133-147. MR 29:1603

6. L. C. Evans Partial regularity for stationary harmonic maps into spheres, Arch. Rational Mech. Anal. 116 (1991), 101-113. MR 93m:58026

7. M. Feldman Partial regularity for harmonic maps of evolution into spheres, preprint (1994).

8. F. Hélein Régularité des applications faiblement harmoniques entre une surface et une sphere, C. R. Acad. Sci. Paris. Sér I Math. 311 (1990), 519-524. MR 92a:58034

9. __ Régularité des applications faiblement harmoniques entre une surface et une variété riemannienne, C. R. Acad. Sci. Paris Sér. I Math. 312 (1991), 591-596. MR 92e:58055

10. J. Moser A Harnack inequality for parabolic differential equations, Comm. Pure Appl. Math. 17 (1964), 101-134. MR 28:2357

11. L. Mou Removability of singular sets of harmonic maps, preprint, 1993.

12. R. Schoen Analytical aspects of the harmonic map problem, Seminar on Nonlinear Partial Differential Equations (S. S. Chern, ed.), vol. 2, Math. Sci. Res. Inst. Publ., Springer-Verlag, New York, Berlin, Heidelberg, and Tokyo, 1984, pp. 321-358. MR 86b:58032

13. L. Simon Singularities of geometric variational problems, Graduate Summer School Lecture at Stanford, 1993.

Department of Mathematics, University of Florida, Gainesville, Florida 32611

E-mail address: yunmei@math.ufl.edu

E-mail address: flaminio@math.ufl.edu 